\title{
The rational topology of gauge groups and of spaces of connections
}

\author{
Svjetlana Terzić \\ To the memory of my teacher and friend, Professor Yuri Petrovich Solovyov
}

\begin{abstract}
Let $P$ be a principal bundle with semisimple compact simply connected structure group $G$ over a compact simply connected four-manifold $M$. In this paper we give explicit formulas for the rational homotopy groups and cohomology algebra of the gauge group and of the space of (irreducible) connections modulo gauge transformations for any such bundle.
\end{abstract}

\section{Introduction}

Our aim in this paper is to compute the rational homotopy groups and the rational cohomology of the gauge group and of the space of connections modulo gauge transformations for principal bundles over four-manifolds. We assume that $G$ is a semisimple compact simply connected Lie group and that $M$ is compact and simply connected.

Note that in some particular cases some of these computations have already been done. Namely, Donaldson [Don86], whose original calculation precedes the book [DK90], computed the cohomology structure of the quotients of spaces of connections for SU(2)-principal bundles over compact simply connected four-manifolds, but the proof essentially uses the fact that the structure group is $\mathrm{SU}(2)$.

We propose here a general approach which appeals to Sullivan's minimal model theory. We proceed as follows. First we compute the rational homotopy groups of the gauge group using the result of Singer [Sin78] characterising the weak homotopy type of base-point-preserving gauge groups, and the Whitehead-Milnor theorem [Mil58, Whi49] giving the homotopy type of a simply connected four-manifold $M$. Then the corresponding fibrations between the gauge groups and spaces of (irreducible) connections yield the rational homotopy groups of the quotients.

Having computed the rational homotopy groups of the quotients of spaces of connections, the nilpotency of the space of connections modulo base point gauge transformations group will make it possible to apply Sullivan's minimal model theory for the cohomology computation. Since it will turn out that these spaces have free cohomology algebras, it will immediately imply that they are formal in the sense of Sullivan.

In the case of the gauge groups, the application of the Sullivan minimal model theory is possible because they are $H$-spaces.

As examples we recover the calculation of the rational cohomology in the case when $G=\mathrm{SU}(2)$ and also provide the calculation in the case when $G=\mathrm{E}_{8}$.

Received 22 February 2003, accepted in final form 17 November 2003, published online 1 December 2004.

2000 Mathematics Subject Classification 55P62, 57R19, 58B05, 81T13.

Keywords: rational topology, gauge groups, spaces of connections.

The results in this paper were partially obtained while the author was postdoctorally supported by the $D F G$ Graduiertenkolleg 'Mathematik im Bereich ihrer Wechselwirkung mit der Physik' at the Mathematical Institute of the Ludwig-Maximilian University in Munich.

This journal is (c) Foundation Compositio Mathematica 2005. 


\section{RATiONAL TOPOLOGY OF GAUGE GROUPS}

\subsection{General remarks}

In this section we briefly recall some general facts on the topology of gauge groups and spaces of connections. For definitions and detailed information on this topic we refer to [DK90].

Let $\pi: P \rightarrow M$ be a $G$-principal bundle, where $G$ is a semisimple compact simply connected Lie group and $M$ a compact simply connected four-manifold. Let $\mathcal{G}$ be the group of gauge transformations of this principal bundle which induce the identity on the base.

Let $\mathcal{A}$ denote the space of all connections and $\mathcal{A}^{*}$ the subspace of all irreducible connections on the bundle $P$. We assume that $\mathcal{A}$ and $\mathcal{G}$ are equipped with certain Sobolev topologies. Usually one fixes the Sobolev class $L_{p-1}^{2}$ for $\mathcal{A}$ and $L_{p}^{2}$ for $\mathcal{G}$, but since we will be interested only in homotopyinvariant properties, the particular choice of $p$ is not important, as long as $p$ is large enough.

The action of $\mathcal{G}$ on $\mathcal{A}$ and $\mathcal{A}^{*}$ is not free in general. In order to have a free action on $\mathcal{A}$, it turns out that one should consider those gauge transformations which fix one fiber. Such automorphisms of $P$ we denote by $\mathcal{G}_{0}$. Besides that, one also has the free action of the group $\tilde{\mathcal{G}}=\mathcal{G} / Z(G)$ on $\mathcal{A}^{*}$, where by $Z(G)$ we denote the center of the group $G$. Note that $Z(G)$ is finite, since we assume $G$ to be semisimple.

The corresponding quotients we denote $\tilde{\mathcal{B}}=\mathcal{A} / \mathcal{G}_{0}, \tilde{\mathcal{B}}^{*}=\mathcal{A}^{*} / \mathcal{G}_{0}$ and $\mathcal{B}^{*}=\mathcal{A}^{*} / \tilde{\mathcal{G}}$. Since in all these cases we have free actions, one gets the corresponding fibrations

$$
\begin{gathered}
\mathcal{G}_{0} \rightarrow \mathcal{A} \rightarrow \tilde{\mathcal{B}}, \\
\mathcal{G}_{0} \rightarrow \mathcal{A}^{*} \rightarrow \tilde{\mathcal{B}}^{*}, \\
\tilde{\mathcal{G}} \rightarrow \mathcal{A}^{*} \rightarrow \mathcal{B}^{*} .
\end{gathered}
$$

We are interested in the rational topology of these objects, i.e. firstly in a computation of their rational homotopy groups, and secondly in a computation of their rational cohomology.

For these purposes the following observations are useful (see [DK90]).

\subsection{Remarks}

i) $\mathcal{A}$ is contractible, since it is an affine space.

ii) $\pi_{j}\left(\mathcal{A}^{*}\right)=0$ as $\mathcal{A}^{*}$ is weakly homotopy equivalent to $\mathcal{A}$.

iii) $\tilde{\mathcal{B}}^{*}$ is weakly homotopy equivalent to $\tilde{\mathcal{B}}$.

iv) $\tilde{\mathcal{G}}=\mathcal{G} / Z(G)$ and hence we have a fibration $Z(G) \rightarrow \mathcal{G} \rightarrow \tilde{\mathcal{G}}$ which gives an exact homotopy sequence

$$
\cdots \rightarrow \pi_{j}(Z(G)) \rightarrow \pi_{j}(\mathcal{G}) \rightarrow \pi_{j}(\tilde{\mathcal{G}}) \rightarrow \pi_{j-1}(Z(G)) \rightarrow \cdots
$$

Since $Z(G)$ is finite we have that, $\pi_{j}(Z(G))=0$ for $j \geqslant 1$ and, therefore,

$$
\pi_{j}(\mathcal{G})=\pi_{j}(\tilde{\mathcal{G}}) \text { for } j \geqslant 2 .
$$

For $j=1$ we have the exact sequence

$$
0 \rightarrow \pi_{1}(\mathcal{G}) \rightarrow \pi_{1}(\tilde{\mathcal{G}}) \rightarrow Z(G) \rightarrow \pi_{0}(\mathcal{G}) \rightarrow \pi_{0}(\tilde{\mathcal{G}}) .
$$

v) $G=\mathcal{G} / \mathcal{G}_{0}$ and we have also the fibration $\mathcal{G}_{0} \rightarrow \mathcal{G} \rightarrow G$, which implies the exact sequence

$$
\pi_{j+1}(G) \rightarrow \pi_{j}\left(\mathcal{G}_{0}\right) \rightarrow \pi_{j}(\mathcal{G}) \rightarrow \pi_{j}(G) .
$$

\subsection{Algebraic topology tools}

We recall here some well known facts from algebraic topology which will be useful for our purposes (see $[$ Swi75]). 


\section{S. TERZIĆ}

Let $M=X \cup_{f} C Y$ be a cofibration, i.e. intuitively we glue the base of the cone $C Y$ to $X$ by means of $f$. Denote by $S X$ the suspension of $X$ which we may regard as being the quotient $(I \times X) /\left(\{0\} \times X \cup I \times\left\{x_{0}\right\} \cup\{1\} \times X\right)$. Then the sequence

$$
\begin{gathered}
(Y, *) \stackrel{f}{\rightarrow}(X, *) \stackrel{j}{\rightarrow}(M, *) \stackrel{k^{\prime}}{\rightarrow}(S Y, *) \stackrel{S f}{\rightarrow}(S X, *) \stackrel{S j}{\rightarrow}(S M, *) \rightarrow \cdots \\
\quad \ldots \stackrel{S^{n} f}{\rightarrow}\left(S^{n} Y, *\right) \stackrel{S^{n} f}{\rightarrow}\left(S^{n} X, *\right) \stackrel{S^{n} j}{\rightarrow}\left(S^{n} M, *\right) \rightarrow \cdots
\end{gathered}
$$

is coexact. This means that the mapping functor $\operatorname{Map}(-, G)$ will turn this sequence into an exact sequence

$$
[Y ; G] \stackrel{f^{*}}{\leftarrow}[X ; G] \stackrel{j^{*}}{\leftarrow}[M ; G] \stackrel{k^{\prime *}}{\leftarrow}[S Y ; G] \stackrel{S f^{*}}{\leftarrow}[S X ; G] \stackrel{S j^{*}}{\leftarrow}[S M ; G] \leftarrow \cdots
$$

The following obvious observation will be useful for our further computation.

LEMMA 1.

$$
\pi_{j}\left(\operatorname{Map}_{*}(M, G)\right)=\left[S^{j} M ; G\right], \quad j \in \mathbb{N} .
$$

\section{Rational homotopy groups of gauge groups and of spaces of connections}

Let $M$ be a compact simply connected four-dimensional manifold. Then, by the result of Whitehead and Milnor [Mil58, Whi49], we know that $M$ is homotopically a cofibration, i.e. $M=$ $\bigvee_{b_{2}(M)} S^{2} \cup_{h} D^{4}$. It means that, up to homotopy, $M$ is obtained by attaching a four-cell to the wedge of two-spheres by an attaching map $h: S^{3} \rightarrow \bigvee_{b_{2}(M)} S^{2}$. Since $D^{4}=C S^{3}$ and $S S^{k}=S^{k+1}$, we can apply what was said in $\S 1.3$ to this cofibration and get the following exact sequence:

$$
\begin{aligned}
{\left[S^{3} ; G\right] } & \leftarrow\left[\bigvee_{b_{2}(M)} S^{2} ; G\right] \leftarrow[M ; G] \leftarrow\left[S^{4} ; G\right] \leftarrow\left[\bigvee_{b_{2}(M)} S^{3} ; G\right] \leftarrow \cdots \\
& \leftarrow\left[S^{n+3} ; G\right] \leftarrow\left[\bigvee_{b_{2}(M)} S^{n+2} ; G\right] \leftarrow\left[S^{n} M ; G\right] \leftarrow \cdots,
\end{aligned}
$$

which is actually

$$
\begin{aligned}
\pi_{3}(G) & \leftarrow \bigoplus_{b_{2}(M)} \pi_{2}(G) \leftarrow[M ; G] \leftarrow \pi_{4}(G) \leftarrow \bigoplus_{b_{2}(M)} \pi_{3}(G) \leftarrow \cdots \\
& \leftarrow \pi_{n+3}(G) \leftarrow \bigoplus_{b_{2}(M)} \pi_{n+2}(G) \leftarrow\left[S^{n} M ; G\right] \leftarrow \cdots
\end{aligned}
$$

We are able to compute first the rational homotopy groups of $\mathcal{G}_{0}$ because of the following theorem of Singer [Sin78], which, among other things, proves that the weak homotopy type of $\mathcal{G}_{0}$ is independent of $P$.

Theorem 1. If $G$ is a compact simply connected semisimple Lie group, then for $\operatorname{dim} M \leqslant 4$ we have that

$$
\mathcal{G}_{0} \sim \operatorname{Map}_{*}(M, G)
$$

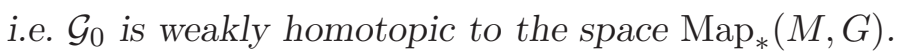

In particular, Theorem 1 gives that in the case of four-dimensional manifolds we have that

$$
\pi_{j}\left(\mathcal{G}_{0}\right)=\pi_{j}\left(\operatorname{Map}_{*}(M, G)\right),
$$

for any $j \in \mathbb{N}$.

Let us first recall some well known facts on the rational cohomology of connected compact Lie groups. 


\section{RATiONAL TOPOLOGY OF GAUGE GROUPS}

Remark 1. The classical Hopf theorem [Bor53] gives that the cohomology algebra of a compact connected Lie group is an exterior algebra over odd-degree generators, i.e. $H^{*}(G)=\bigwedge\left(z_{1}, \ldots, z_{n}\right)$, where $n=\operatorname{rk} G$ and $\operatorname{deg} z_{i}=2 k_{i}-1$. Here $\operatorname{rk} G$ is the rank of the group $G$, i.e. the dimension of the maximal torus in $G$, and the numbers $k_{i}, 1 \leqslant i \leqslant n$, are so-called exponents of the group $G$. Because of formality, rational homotopy theory then gives that $\operatorname{rk} \pi_{2 k}(G)=0$, that $\operatorname{rk} \pi_{2 k-1}(G)=0$ if $k$ is not an exponent for $G$, while for a $k$ being an exponent for $G$ we have that $\operatorname{rk} \pi_{2 k-1}(G)=$ $b_{2 k-1}(G)=\nu(k)$. Here by $\nu(k)$ we denote the multiplicity of the exponent $k$. Obviously, it is satisfied that $\operatorname{rk} G=\sum_{j \in \mathbb{N}} \operatorname{rk} \pi_{j}(G)$.

Remark 2. Since $\pi_{2 j}(G)$ are finite, Theorem 1 and the sequence (8) imply that $\pi_{0}\left(\mathcal{G}_{0}\right)$ is finite.

Remark 3. Since we are interested in the rational homotopy groups of these spaces, we need to consider the sequence $(8)$ tensored by $\mathbb{Q}$. Since $\pi_{1}\left(\mathcal{G}_{0}\right)$ is abelian, we can also tensor $\pi_{1}\left(\mathcal{G}_{0}\right)$ by $\mathbb{Q}$. Namely, $\mathcal{G}_{0}$ is a topological group and, thus, all its connected components are homeomorphic, so we can fix the component $\mathcal{G}_{0}^{e}$ corresponding to the identity automorphism. It is obviously also a topological group and, thus, homotopically simple. ${ }^{1}$

Proposition 1. The ranks of the homotopy groups of the group $\mathcal{G}_{0}$ are given by

$$
\operatorname{rk} \pi_{j}\left(\mathcal{G}_{0}\right)=b_{2}(M) \operatorname{rk} \pi_{j+2}(G)+\operatorname{rk} \pi_{j+4}(G), \quad j \in \mathbb{N} .
$$

Proof. Applying Theorem 1 to the sequence (8) we have that

$$
\cdots \leftarrow \pi_{j+3}(G) \otimes \mathbb{Q} \leftarrow \bigoplus_{b_{2}(M)} \pi_{j+2}(G) \otimes \mathbb{Q} \leftarrow \pi_{j}\left(\mathcal{G}_{0}\right) \otimes \mathbb{Q} \leftarrow \pi_{j+4}(G) \otimes \mathbb{Q} \cdots
$$

Since for $j$ even we know that $\pi_{j}(G) \otimes \mathbb{Q}=0$, the sequence (11) immediately implies that $\pi_{j}\left(\mathcal{G}_{0}\right) \otimes \mathbb{Q}=0$ for $j$ even.

Therefore, we will assume that $j$ is odd. Then from the sequence (11) we can extract the following short exact sequence

$$
0 \leftarrow b_{2}(M) \pi_{j+2}(G) \otimes \mathbb{Q} \leftarrow \pi_{j}\left(\mathcal{G}_{0}\right) \otimes \mathbb{Q} \leftarrow \pi_{j+4}(G) \otimes \mathbb{Q} \leftarrow 0 .
$$

This gives that

$$
\operatorname{rk} \pi_{j}\left(\mathcal{G}_{0}\right)=b_{2}(M) \operatorname{rk} \pi_{j+2}(G)+\operatorname{rk} \pi_{j+4}(G)
$$

Proposition 2. The ranks of the homotopy groups of the group $\mathcal{G}$ are given by

$$
\operatorname{rk} \pi_{j}(\mathcal{G})=b_{2}(M) \operatorname{rk} \pi_{j+2}(G)+\operatorname{rk} \pi_{j+4}(G)+\operatorname{rk} \pi_{j}(G), \quad j \in \mathbb{N} .
$$

Proof. For the same reasons as in the case of the group $\mathcal{G}_{0}$ we are able to tensor the sequence (5) by $\mathbb{Q}$. Thus, tensoring the sequence $(5)$ by $\mathbb{Q}$ we get the exact sequence

$$
\pi_{j+1}(G) \otimes \mathbb{Q} \rightarrow \pi_{j}\left(\mathcal{G}_{0}\right) \otimes \mathbb{Q} \rightarrow \pi_{j}(\mathcal{G}) \otimes \mathbb{Q} \rightarrow \pi_{j}(G) \otimes \mathbb{Q} \rightarrow \pi_{j-1}\left(\mathcal{G}_{0}\right) \otimes \mathbb{Q} .
$$

First, for $j$ even, this sequence implies that $\pi_{j}(\mathcal{G}) \otimes \mathbb{Q}=0$.

Therefore we assume $j$ to be odd. Then the sequence (14) and Proposition 1 give the following short exact sequence:

$$
0 \rightarrow \pi_{j}\left(\mathcal{G}_{0}\right) \otimes \mathbb{Q} \rightarrow \pi_{j}(\mathcal{G}) \otimes \mathbb{Q} \rightarrow \pi_{j}(G) \otimes \mathbb{Q} \rightarrow 0
$$

This implies that

$$
\begin{aligned}
\operatorname{rk} \pi_{j}(\mathcal{G}) & =\operatorname{rk} \pi_{j}(G)+\operatorname{rk} \pi_{j}\left(\mathcal{G}_{0}\right) \\
& =b_{2}(M) \operatorname{rk} \pi_{j+2}(G)+\operatorname{rk} \pi_{j+4}(G)+\operatorname{rk} \pi_{j}(G)
\end{aligned}
$$

\footnotetext{
${ }^{1}$ Meaning that its fundamental group is abelian and acts trivially on its higher homotopy groups.
} 


\section{S. TERZIĆ}

Remark 4. Since we assume $G$ to be semisimple, the sequences (4) and (5) give that for all $j \in \mathbb{N}$

$$
\pi_{j}(\tilde{\mathcal{G}}) \otimes \mathbb{Q}=\pi_{j}(\mathcal{G}) \otimes \mathbb{Q}
$$

and by Proposition 2 we have computed the rational homotopy groups for $\tilde{\mathcal{G}}$.

COROLlary 1. The rational homotopy groups for $\tilde{\mathcal{B}}, \tilde{\mathcal{B}}^{*}$ and $\mathcal{B}^{*}$ are given by

i) $\operatorname{rk} \pi_{j}(\tilde{\mathcal{B}})=\operatorname{rk} \pi_{j}\left(\tilde{\mathcal{B}}^{*}\right)=b_{2}(M) \operatorname{rk} \pi_{j+1}(G)+\operatorname{rk} \pi_{j+3}(G), j \geqslant 1$,

ii) $\operatorname{rk} \pi_{j}\left(\mathcal{B}^{*}\right)=b_{2}(M) \operatorname{rk} \pi_{j+1}(G)+\operatorname{rk} \pi_{j+3}(G)+\operatorname{rk} \pi_{j-1}(G), j \geqslant 1$.

Proof. Since the homotopy groups of the total spaces in the fibrations (1), (2) and (3) are trivial we get that

$$
\begin{gathered}
\pi_{j}(\tilde{\mathcal{B}}) \otimes \mathbb{Q}=\pi_{j}\left(\tilde{\mathcal{B}}^{*}\right) \otimes \mathbb{Q}=\pi_{j-1}\left(\mathcal{G}_{0}\right) \otimes \mathbb{Q}, \\
\pi_{j}\left(\mathcal{B}^{*}\right) \otimes \mathbb{Q}=\pi_{j-1}(\tilde{\mathcal{G}}) \otimes \mathbb{Q} .
\end{gathered}
$$

Now, Propositions 1 and 2 and Remark 4 give the statement.

\section{Rational cohomology of gauge groups and of spaces of connections}

\subsection{Rational cohomology of gauge groups}

As we already said in the proof of Proposition 2, the identity component $\mathcal{G}^{e}$ of the gauge group $\mathcal{G}$ is a homotopically simple space. By Proposition 2 it is of finite type and has only odd-degree nontrivial rational homotopy groups.

Now we can apply Sullivan's minimal model theory, since, more generally, for nilpotent spaces ${ }^{2}$ of finite type it works well. Namely, for a nilpotent space $X$ of finite type, minimal model theory gives that the degrees of and the numbers of generators in its minimal model are given by its nontrivial rational homotopy groups (see [Leh77]). More precisely, if $\mu(X)$ denotes the minimal model for $X$ then the number of its generators $\mu_{j}(X)$ of degree $j$ is equal to $\operatorname{rk} \pi_{j}(X)$.

In our case this gives that the minimal model for $\mathcal{G}^{e}$ has only odd-degree generators. On the other hand, for $H$-spaces of finite type the Hopf theorem [Bor53] implies that their cohomology algebra is a free commutative algebra. Therefore, $H^{*}\left(\mathcal{G}^{e}\right)$ is an exterior algebra of odd-degree generators. In particular this gives that $\mathcal{G}^{e}$ is formal in the sense of Sullivan and its minimal model coincides with its cohomology algebra. Moreover, $H^{*}\left(\mathcal{G}^{e}\right)$ has generators of degree $2 j-1$ if and only if rk $\pi_{2 j-1}(\mathcal{G}) \neq 0$.

Obviously, the cohomology algebra $H^{*}(\mathcal{G})$ is equal to the sum of $\left|\pi_{0}(\mathcal{G})\right|$ copies of $\mathcal{G}^{e}$, i.e.

$$
H^{*}(\mathcal{G})=\bigoplus_{\left|\pi_{0}(\mathcal{G})\right|} H^{*}\left(\mathcal{G}^{e}\right) .
$$

Remark 5. If for the structure group $G$ we have that $\pi_{4}(G)=0$, then the sequence (8) implies that $\mathcal{G}_{0}$ is connected. That is the case, say, when $G=\mathrm{SU}(n), n \geqslant 3, G=\operatorname{Spin}(n), n \geqslant 6$, or $G$ is a simply connected Lie group of exceptional type. Obviously, the same is true for the groups $\mathcal{G}$ and $\mathcal{G}_{0}$.

Equation (16) and Proposition 2 immediately give the following theorem.

THEOREM 2. If $G$ is a compact simply connected semisimple Lie group, then $H^{*}\left(\mathcal{G}^{e}\right)$ is an exterior algebra in $\left(b_{2}(M)+2\right) \mathrm{rk} G-1$ odd-degree generators. The number of generators of degree $j$ is equal to $b_{2}(M) \operatorname{rk} \pi_{j+2}(G)+\operatorname{rk} \pi_{j+4}(G)+\operatorname{rk} \pi_{j}(G)$.

\footnotetext{
${ }^{2}$ They are given by the condition that the fundamental group is nilpotent and acts nilpotently on higher homotopy groups.
} 


\section{RATiONAL TOPOLOGY OF GAUGE GROUPS}

\subsection{Rational cohomology of $\tilde{\mathcal{B}}$ and of $\tilde{\mathcal{B}}^{*}$}

Theorem 3. The rational cohomology algebra of $\tilde{\mathcal{B}}$ is a polynomial algebra in $\left(b_{2}(M)+1\right) \mathrm{rk} G-1$ generators of even degree. The number of generators of degree $j$ is equal to

$$
b_{2}(M) \operatorname{rk} \pi_{j+1}(G)+\operatorname{rk} \pi_{j+3}(G) .
$$

We give a general proof which works for any semisimple compact simply connected Lie group $G$. As we point out in Remark 7 below, under some additional assumptions on the group $G$, the proof is much simpler.

Proof of Theorem 3. By the result of [AB82] and [Don86], we know that $\tilde{\mathcal{B}}$ has the weak homotopy type of $\operatorname{Map}_{*}\left(M, B_{G}\right)_{P}$, where the latter denotes the homotopy class of $\operatorname{Map}_{*}\left(M, B_{G}\right)$ corresponding to the maps inducing the bundle $P$. Since $M$ and $B_{G}$ are connected, the result of Hilton [Hil84] implies that any connected component of the $\operatorname{space}^{\operatorname{Map}_{*}}\left(M, B_{G}\right)$ is nilpotent. Hence, $\operatorname{Map}_{*}\left(X, B_{G}\right)_{P}$ is also nilpotent. As $\tilde{\mathcal{B}}$ is weakly homotopy equivalent to $\operatorname{Map}_{*}\left(X, B_{G}\right)_{P}$, it implies that $\tilde{\mathcal{B}}$ is also nilpotent. Moreover, by Corollary 1 we know that $\tilde{\mathcal{B}}$ is of finite type, so we can apply Sullivan's minimal model theory. It gives that for all $j$

$$
\operatorname{rk} \pi_{j}(\tilde{\mathcal{B}})=\operatorname{dim}(\mu(\tilde{\mathcal{B}}))_{j},
$$

and thus

$$
\mu(\tilde{\mathcal{B}})=\mathbb{Q}\left[x_{1}, \ldots, x_{p}\right],
$$

where $x_{i}$ correspond to the nontrivial rational homotopy groups of $\tilde{\mathcal{B}}$. Since, by Corollary 1 , $\pi_{j}(\tilde{\mathcal{B}}) \otimes \mathbb{Q}=0$ for $j$ odd, we have that all $x_{i}$ are of even degree. This implies that the differential in this minimal model has to be zero. Since by the definition of the minimal model we have that $H^{*}(\mu(\tilde{\mathcal{B}})) \cong H^{*}(\tilde{\mathcal{B}})$, it follows that

$$
H^{*}(\tilde{\mathcal{B}}, \mathbb{Q})=\mathbb{Q}\left[x_{1}, \ldots, x_{p}\right] .
$$

By Corollary 1 the number of generators of degree $j(j \geqslant 1)$ is $b_{2}(M) \operatorname{rk} \pi_{j+1}(G)+\operatorname{rk} \pi_{j+3}(G)$. Then, if we sum these numbers, we get that the number of generators in $H^{*}(\tilde{\mathcal{B}}, \mathbb{Q})$ is $\left(b_{2}(M)+1\right)$ rk $G-1$.

Since Theorem 3 gives that $H^{*}(\tilde{\mathcal{B}})$ is a free algebra, it immediately implies the following corollary. Corollary 2. The spaces $\tilde{\mathcal{B}}$ and $\tilde{\mathcal{B}}^{*}$ are formal in the sense of Sullivan.

Remark 6 . We pointed out in $\S 1.2$ that $\tilde{\mathcal{B}}^{*}$ is weakly homotopy equivalent to $\tilde{\mathcal{B}}$, hence they have the same rational cohomology.

Remark 7 . If for the structure group $G$ we have that $\pi_{4}(G)=0$, then from Remark 5 and the fibrations (1) and (2) it follows that the spaces $\tilde{\mathcal{B}}$ and $\tilde{\mathcal{B}}^{*}$ are simply connected. Therefore it follows immediately that the generators in the minimal models for $\tilde{\mathcal{B}}$ and $\tilde{\mathcal{B}}^{*}$ are given by their nontrivial rational homotopy groups.

\subsection{Rational cohomology of $\mathcal{B}^{*}$}

Theorem 4. The rational cohomology algebra of $\mathcal{B}^{*}$ is a polynomial algebra in even-degree $\left(b_{2}(M)+2\right) \mathrm{rk} G-1$ generators. The number of generators of degree $j$ is equal

$$
b_{2}(M) \operatorname{rk} \pi_{j+1}(G)+\operatorname{rk} \pi_{j+3}(G)+\operatorname{rk} \pi_{j-1}(G) .
$$

Proof. We can get $\mathcal{B}^{*}$ as a base of the principal fibration $\tilde{\mathcal{B}}^{*} \rightarrow \mathcal{B}^{*}$ with a fiber $G / Z(G)$. Note that, since $Z(G)$ is finite, the groups $G$ and $G / Z(G)$, and hence their classifying spaces, have the same rational cohomology. Therefore, without loss of generality, we are not going to differentiate between them. 


\section{S. TERZIĆ}

Since we are interested in the cohomology of $\mathcal{B}^{*}$, it turns out to be much easier to apply the Borel construction to this fibration in order to work with the total space of the fibration instead of the base. This goes as follows. Let $\mathrm{E}_{G} \rightarrow B_{G}$ be the universal fibration for the group $G$ and denote by $\tilde{\mathcal{B}}_{G}^{*}$ the quotient $\tilde{\mathcal{B}}_{G}^{*}=\left(\tilde{\mathcal{B}}^{*} \times \mathrm{E}_{G}\right) / G$ given by the diagonal action of the group $G$. Let us consider the Serre fibration

$$
\tilde{\mathcal{B}}_{G}^{*} \rightarrow B_{G}
$$

with a fiber $\tilde{\mathcal{B}}^{*}$. Obviously $\tilde{\mathcal{B}}_{G}^{*}$ is weakly homotopy equivalent to $\mathcal{B}^{*}$.

Let $\mu\left(\tilde{\mathcal{B}}^{*}\right)$ and $\mu\left(B_{G}\right)$ be the minimal models for $\tilde{\mathcal{B}}^{*}$ and $B_{G}$ respectively. The fact that $B_{G}$ is simply connected implies that $\left(\mu\left(\tilde{\mathcal{B}}^{*}\right) \otimes \mu\left(B_{G}\right), d\right)$ is a Sullivan model for $\tilde{\mathcal{B}}_{G}^{*}$ (see [FHT00]). Moreover, since all the generators in $\mu\left(\tilde{\mathcal{B}}^{*}\right)$ and $\mu\left(B_{G}\right)$ are of even degree, it follows that $d=0$ and $\left(\mu\left(\tilde{\mathcal{B}}^{*}\right) \otimes \mu\left(B_{G}\right), d=0\right)$ is the minimal model for $\tilde{\mathcal{B}}_{G}^{*}$ and, hence, for $\mathcal{B}^{*}$. It follows that

$$
H^{*}\left(\mathcal{B}^{*}\right)=H^{*}(\tilde{\mathcal{B}}) \otimes H^{*}\left(B_{G}\right) .
$$

It is a well known fact [Bor53] that $H^{*}\left(B_{G}\right)$ is a polynomial algebra in $\mathrm{rk} G$ generators and the number of its generators of degree $j$ is equal to $\operatorname{rk} \pi_{j}(G)$. Combining this with Theorem 3 , the statement follows.

Since Theorem 4 gives that $H^{*}\left(\mathcal{B}^{*}\right)$ is a free algebra, it implies the following corollary.

Corollary 3. The space $\mathcal{B}^{*}$ is formal in the sense of Sullivan.

Remark 8. Again, if for the structure group $G$ we have that $\pi_{4}(G)=0$, then $\mathcal{B}^{*}$ is simply connected and using Proposition 1 we immediately get its minimal model.

As a direct application of the above results we will get the rational cohomology of the gauge group $\mathcal{G}$ and the proposition proved in [DK90] on rational cohomology of $\tilde{\mathcal{B}}$ and $\mathcal{B}^{*}$ for $\mathrm{SU}(2)$ principal bundles over four-manifolds. We also provide the same calculation when the structure group of the bundle is $\mathrm{E}_{8}$.

Example 1. Let $G=\mathrm{SU}(2)$. It has one exponent $k=2$ of multiplicity 1 and, thus, $\pi_{3}(G) \otimes \mathbb{Q}=\mathbb{Q}$, while all the other rational homotopy groups are trivial. Proposition 1 gives that

$$
\pi_{j}\left(\mathcal{G}_{0}\right) \otimes \mathbb{Q}=0, \quad \text { for } j \geqslant 2, \quad \pi_{1}\left(\mathcal{G}_{0}\right) \otimes \mathbb{Q}=\mathbb{Q}^{b_{2}(M)} .
$$

Further by Proposition 2, we get now that

$$
\pi_{1}(\mathcal{G}) \otimes \mathbb{Q}=\mathbb{Q}^{b_{2}(M)}, \quad \pi_{3}(\mathcal{G}) \otimes \mathbb{Q}=\mathbb{Q}
$$

while all the other rational homotopy groups for $\mathcal{G}$ are trivial. The same is true for $\tilde{\mathcal{G}}$.

Using Corollary 1 we get that the nontrivial rational homotopy groups for $\tilde{\mathcal{B}}$ and $\mathcal{B}^{*}$ are given by

$$
\begin{gathered}
\pi_{2}(\tilde{\mathcal{B}}) \otimes \mathbb{Q}=\mathbb{Q}^{b_{2}(M)}, \\
\pi_{2}\left(\mathcal{B}^{*}\right) \otimes \mathbb{Q}=\mathbb{Q}^{b_{2}(M)}, \quad \pi_{4}\left(\mathcal{B}^{*}\right) \otimes \mathbb{Q}=\mathbb{Q} .
\end{gathered}
$$

Then Theorem 3 says that the cohomology algebra for $\tilde{\mathcal{B}}$ is given by

$$
H^{*}(\tilde{\mathcal{B}})=\mathbb{Q}\left[x_{1}, \ldots, x_{b_{2}(M)}\right], \quad \operatorname{deg} x_{i}=2 .
$$

Theorem 4 gives that the cohomology algebra for $\mathcal{B}^{*}$ is

$$
H^{*}\left(\mathcal{B}^{*}\right)=\mathbb{Q}\left[x_{1}, \ldots, x_{b_{2}(M)}, y\right], \quad \operatorname{deg} x_{i}=2, \operatorname{deg} y=4 .
$$

Also, by Theorem 2 we have that

$$
H^{*}\left(\mathcal{G}^{e}\right)=\bigwedge\left(z_{1}, \ldots, z_{b_{2}(M)}, w\right), \quad \operatorname{deg} z_{i}=1, \operatorname{deg} w=3
$$




\section{RATiONAL TOPOLOGY OF GAUGE GROUPS}

Example 2. It is also interesting to consider the case when $G$ is a simply connected exceptional Lie group such as $\mathrm{E}_{8}$. The nontrivial rational homotopy groups for $\mathrm{E}_{8}$ are given by $\pi_{2 k-1}\left(\mathrm{E}_{8}\right) \otimes \mathbb{Q}=\mathbb{Q}$, where $k=2,8,12,14,18,20,24,30$, since these are the exponents for $\mathrm{E}_{8}$. Proposition 1 gives that the nontrivial rational homotopy groups for $\mathcal{G}_{0}$ are given by

$$
\begin{gathered}
\pi_{j}\left(\mathcal{G}_{0}\right) \otimes \mathbb{Q}=\mathbb{Q}, \quad \text { for } j=11,19,23,31,35,43,55 ; \\
\pi_{j}\left(\mathcal{G}_{0}\right) \otimes \mathbb{Q}=\mathbb{Q}^{b_{2}(M)}, \quad \text { for } j=1,13,21,25,33,37,45,57 .
\end{gathered}
$$

Then Proposition 2 gives that the nontrivial rational homotopy groups for $\mathcal{G}$ (and for $\tilde{\mathcal{G}}$ ) are

$$
\begin{gathered}
\pi_{j}(\mathcal{G}) \otimes \mathbb{Q}=\mathbb{Q}, \quad \text { for } j=3,11,15,19,27,31,39,43,47,55,59 ; \\
\pi_{j}(\mathcal{G}) \otimes \mathbb{Q}=\mathbb{Q} \oplus \mathbb{Q}, \quad \text { for } j=23,35 ; \\
\pi_{j}(\mathcal{G}) \otimes \mathbb{Q}=\mathbb{Q}^{b_{2}(M)}, \quad \text { for } j=1,13,21,25,33,37,45,57 .
\end{gathered}
$$

By Corollary 1 we see that the nontrivial rational homotopy groups for $\tilde{\mathcal{B}}$ and $\mathcal{B}^{*}$ are given by

$$
\begin{gathered}
\pi_{j}(\tilde{\mathcal{B}}) \otimes \mathbb{Q}=\mathbb{Q}, \quad \text { for } j=12,20,24,32,36,44,56 ; \\
\pi_{j}(\tilde{\mathcal{B}}) \otimes \mathbb{Q}=\mathbb{Q}^{b_{2}(M)}, \quad \text { for } j=2,14,22,26,34,38,46,58 ; \\
\pi_{j}\left(\mathcal{B}^{*}\right) \otimes \mathbb{Q}=\mathbb{Q}, \quad \text { for } j=4,12,16,20,28,32,40,44,48,56,60 ; \\
\pi_{j}\left(\mathcal{B}^{*}\right) \otimes \mathbb{Q}=\mathbb{Q} \oplus \mathbb{Q}, \quad \text { for } j=24,36 ; \\
\pi_{j}\left(\mathcal{B}^{*}\right) \otimes \mathbb{Q}=\mathbb{Q}^{b_{2}(M)}, \quad \text { for } j=2,14,22,26,34,38,46,58 .
\end{gathered}
$$

Then by Theorem 3, the cohomology algebra $H^{*}(\tilde{\mathcal{B}})$ is a polynomial algebra in $8 b_{2}(M)+7$ generators whose degrees are given by the nontrivial rational homotopy groups for $\tilde{\mathcal{B}}$. Analogously, by Theorem 4 the cohomology algebra $H^{*}\left(\mathcal{B}^{*}\right)$ is a polynomial algebra in $8 b_{2}(M)+15$ generators whose degrees are given by the nontrivial rational homotopy groups for $\mathcal{B}^{*}$. Finally, Theorem 2 gives that the cohomology algebra $H^{*}\left(\mathcal{G}^{e}\right)$ is an exterior algebra in $8 b_{2}(M)+15$ generators whose degrees are also given by the nontrivial rational homotopy groups for $\mathcal{G}$.

Remark 9. Note that the results of this paper do not hold in general if one omits the assumption that the structure group is simply connected. Namely, let us, for example, take $P$ to be a U(3)principal bundle over $S^{4}$. According to [AB82], $\tilde{\mathcal{B}}$ has weak homotopy type of $K(\mathbb{Z} ; 2) \times K(\mathbb{Z} ; 2) \times$ $K(\mathbb{Z} ; 4) \times K(\mathbb{Z} ; 6)$. Therefore, the rational cohomology algebra for $\tilde{\mathcal{B}}$ is given by

$$
H^{*}(\tilde{\mathcal{B}})=\mathbb{Q}\left[x_{1}, x_{2}, y, z\right],
$$

where $\operatorname{deg} x_{1}=\operatorname{deg} x_{2}=2, \operatorname{deg} y=4$ and $\operatorname{deg} z=6$.

On the other hand, Theorem 3 would give that the number of generators in $H^{*}(\tilde{\mathcal{B}})$ is 2 , and, thus, it is not valid in this case. This also shows that, in Theorem 1 of Singer, the condition that the group $G$ is simply connected cannot be omitted.

\section{REFERENCES}

AB82 M. F. Atiyah and R. Bott, The Yang-Mills equations over Riemann surfaces, Philos. Trans. Roy. Soc. London Ser. A 303 (1982), 523-615.

Bor53 A. Borel, Sur la cohomologie des espaces fibrés principaux et des espaces homogènes de groupes de Lie compacts, Ann. of Math. (2) $5 \mathbf{7}$ (1953), 115-207.

DK90 S. K. Donaldson and P. B. Kronheimer, The geometry of four-manifolds (Oxford University Press, Oxford, 1990).

Don86 S. K. Donaldson, Connections, cohomology and the intersection forms on 4-manifolds, J. Differential Geom. 24 (1986), 275-341. 


\section{RATIONAL TOPOLOGY OF GAUGE GROUPS}

FHT00 Y. Felix, S. Halperin and J.-C. Thomas, Rational homotopy theory (Springer, Berlin, 2000).

Hil84 P. J. Hilton, Nilpotente Gruppen und nilpotente Räume, Lecture Notes in Mathematics, vol. 1053 (Springer, Berlin, 1984).

Leh77 D. Lehmann, Théorie homotopique des formes différentielles (d'aprés D. Sullivan), Astérisque 45 (1977).

Mil58 J. Milnor, On simply connected 4-manifolds, in Symposium Internacional de Topologia Algebraica (1958), 122-128.

Sin78 I. M. Singer, Some remarks on the Gribov ambiguity, Commun. Math. Phys. 60 (1978), 7-12.

Swi75 R. M. Switzer, Algebraic topology-homotopy and homology (Springer, Berlin, 1975).

Whi49 J. H. C. Whitehead, On simply connected, 4-dimensional polyhedra, Comment. Math. Helv. 22 (1949), 48-92.

Svjetlana Terzić sterzic@cg.ac.yu

Faculty of Science, University of Montenegro, Cetinjski put bb, 81000 Podgorica, Montenegro, Serbia and Montenegro 